\title{
ISOTOPIES OF HOMEOMORPHISMS OF RIEMANN SURFACES AND A THEOREM ABOUT ARTIN'S BRAID GROUP
}

\author{
BY JOAN S. BIRMAN AND HUGH M. HILDEN \\ Communicated by William Browder, May 18, 1972
}

Let $\tilde{X}, X$ be orientable surfaces. Let $(p, \tilde{X}, X)$ be a regular covering space, possibly branched, with finitely many branch points and a finite group of covering transformations. We require also that every covering transformation leave the branch points fixed. A homeomorphism $\tilde{g}: \widetilde{X} \rightarrow \widetilde{X}$ is said to be "fiber-preserving" with respect to the triplet $(p, \tilde{X}, X)$ if for every pair of points $\tilde{x}, \tilde{x}^{\prime} \in \tilde{X}$ the condition $p(\tilde{x})=p\left(\tilde{x}^{\prime}\right)$ implies $p \tilde{g}(\tilde{x})=p \tilde{g}\left(\tilde{x}^{\prime}\right)$. If $\tilde{g}$ is fiber-preserving and isotopic to the identity map via an isotopy $\tilde{g}_{s}$, then $\tilde{g}$ is said to be "fiber-isotopic to 1" if, for every $s \in[0,1]$, the homeomorphism $\tilde{g}_{s}$ is fiber-preserving.

The condition that an isotopy be a fiber-isotopy imposes a symmetry which one feels, intuitively, is very restrictive. However, we find

THEOREM 1. Let $g: \tilde{X} \rightarrow \widetilde{X}$ be a fiber-preserving homeomorphism which is isotopic to the identity map. If the covering is branched, assume $\tilde{X}$ is not the closed sphere or torus. Then $g$ is fiber-isotopic to the identity.

Theorem 2 expresses a weaker result, which is true without exception.

THEOREM 2. Let $\tilde{g}: \tilde{X} \rightarrow \tilde{X}$ be a fiber-preserving homeomorphism which is isotopic to the identity map. Then its projection $g$ to $X$ is also isotopic to the identity map; however, the isotopy may move branch points.

A special case of Theorem 1 was established by the authors in an earlier paper [1] for the particular situation where $X$ is a 2-sphere, and $\tilde{X}$ is a 2-sheeted covering of $X$ with $2 g+2$ branch points. The proof given here is considerably simpler than the version in [1], and at the same time it holds in a much more general situation. The major tool that made this possible was the device of lifting maps to the universal covering space. The analogous problem in higher-dimensional manifolds has also been studied by the authors, and will be reported on separately.

Let $H(\tilde{X})$ be the group of all orientation-preserving homeomorphisms of $\tilde{X} \rightarrow \tilde{X}$, and let $D(\widetilde{X})$ be the subgroup of those homeomorphisms which are isotopic to the identity map. Let $M(\tilde{X})$ be the quotient group $H(\tilde{X}) / D(\widetilde{X})$, that is the mapping class group of $\widetilde{X}$. Assume that the

AMS 1970 subject classifications. Primary 57A05, 58D99, 20F05, 30A60; Secondary 30A48, 57A10. 
covering $(p, \tilde{X}, X)$ is branched. Let $\widetilde{P}_{1}, \ldots, \widetilde{P}_{n}$ denote the set of branch points, and let $P_{i}=p\left(\widetilde{P}_{i}\right), \quad i=1, \ldots, n$. The branched covering has an associated unbranched covering which we denote by $\left(\tilde{p}, \tilde{X}-\widetilde{P}_{1}, \ldots\right.$, $\left.\widetilde{P}_{n}, X-P_{1}, \ldots, P_{n}\right)$, where $\tilde{p}$ is the restriction of $p$ to $\tilde{X}-\widetilde{P}_{1}, \ldots, \widetilde{P}_{n}$. Let $M_{p}(\tilde{X})$ be the "symmetric" mapping class group of $\tilde{X}$, that is the subgroup of those elements in $M(\tilde{X})$ which can be represented by fiberpreserving maps. Theorem 1 can be rephrased: " $M_{p}(\widetilde{X})$ is canonically isomorphic to $M_{p}\left(\tilde{X}-\widetilde{P}_{1} \cup \ldots \cup \widetilde{P}_{n}\right)$."

Let $T_{g, n}$ denote a Riemann surface of genus $g$ with $n$ points removed. The groups $M\left(T_{g, 0}\right)$ can be expected to play an important role in understanding the topology of 3 manifolds, in Teichmüller theory, and again in the theory of automorphisms of infinite groups. However, for the cases $g \geqq 3$, very little is known about these groups. As a step in this direction, we ask how the subgroups $M_{p}\left(T_{g, 0}\right) \subset M\left(T_{g, 0}\right)$ can be characterized algebraically. Restricting our attention to the case where the group of covering transformations is generated by a single element $t$ of order $k$, we observe that a homeomorphism $h: T_{g, 0} \rightarrow T_{g, 0}$ is fiber-preserving iff $\tilde{h} \tilde{h}^{-1}=\tilde{t}^{s}$ for some $1 \leqq s \leqq k-1$. Hence the subgroup $M_{p}\left(T_{g, 0}\right)$ is necessarily included in the normalizer of the cyclic subgroup $T$ generated by the isotopy class $[t]$ of the element $t$. To determine whether $M_{p}\left(T_{g, 0}\right)$ coincides with the normalizer of $T$, we first show

THEOREM 3. Let $[\mathfrak{t}],[\tilde{h}]=M\left(T_{g, 0}\right)$, where $[\tilde{t}]$ has finite order $k$. Suppose that $[\tilde{h}]$ belongs to the normalizer of $[\tilde{t}]$, i.e.,

$$
[\tilde{h}][\tilde{t}][\tilde{h}]^{-1}=[\mathfrak{t}]^{s}, \quad 1 \leqq s \leqq k-1,(s, k)=1 .
$$

Then $[\tilde{h}]$ and $[\tilde{t}]$ can be represented by topological mappings $\tilde{h}, \tilde{t}$ which have the properties

$$
\tilde{h} \tilde{h}^{-1}=\tilde{t}^{s} \text { and } \tilde{t}^{k}=1 .
$$

Using Theorem 3, we were then able to establish that for the case where the group $T$ is cyclic:

THEOREM 4. The symmetric subgroups $M_{p}\left(T_{g, 0}\right)$ are precisely the normalizers of the cyclic subgroups $T$ generated by any element $[\tau]$ of finite order in $M\left(T_{g, 0}\right)$.

To apply Theorem 4, we restrict our attention to the case of $k$-sheeted cyclic coverings $\left(p, T_{g, 0}, T_{0,0}\right)$ of the sphere $T_{0,0}$ by the closed surface $T_{g, 0}$. In this situation, the covering will have $n$ branch points, where $k, n$ and $g$ are related by the formula $2 g=(k-1)(n-2)$.

For this special case we find

THEOREM 5. Projecting fiber-preserving homeomorphisms induces an isomorphism $i$ between the groups $M_{p}\left(T_{g, 0}\right) / T$ and $M\left(T_{0, n}\right)$. 
Since generators and defining relations are known for the group $M\left(T_{0, n}\right)$ for every integer $n$ (see [3], [4]) we can use this result (which is constructive) to determine explicit presentations for all of the groups $M_{p}\left(T_{g, 0}\right)$.

For the special case $g=2, k=2, n=6$, it was shown in [1] that the group $M_{p}\left(T_{2,0}\right)$ coincides with the full mapping class group $M\left(T_{2,0}\right)$. We find that this situation was special indeed, and, in fact,

THEOREM 6. If $g \geqq 3$, there does not exist any finite cyclic covering with the property that $M_{p}\left(T_{g, 0}\right)$ coincides with $M\left(T_{g, 0}\right)$.

As an application of the above results, we discuss and settle a conjecture about Artin's braid group $B_{n}$. The braid group can be defined as that group of automorphisms of a free group $F_{n}=\left\langle x_{1}, \ldots, x_{n}\right\rangle$ of rank $n$ which maps every generator $x_{i}$ into a conjugate of itself, and preserves the product $x_{1} x_{2} \cdots x_{n}$ [4]. Let $k$ be any integer $\geqq 2$, and let $N_{k}$ be the normal closure in $F_{n}$ of the $n$ elements $x_{1}^{k}, \ldots, x_{n}^{k}$. Then the elements in $B_{n}$ induce a group of automorphisms of $F_{n} / N_{k}$, which we denote by $B_{n, k}$. We show that our geometric results imply

THEOREM 7. $B_{n, k}$ is canonically isomorphic to $B_{n}$.

A detailed report giving proofs of the theorems stated above will be published in another journal. The methods used were a combination of geometric and algebraic arguments and also, in the case of Theorem 3, techniques in Teichmüller theory, similar to those used in [2].

\section{REFERENCES}

1. J. Birman and H. Hilden, Mapping class groups of closed surfaces as covering spaces, Advances in the Theory of Riemann Surfaces, Ann. of Math. Studies, no. 66, Princeton Univ. Press, Princeton, N.J., 1971.

2. A. M. Macbeath, Geometric realization of isomorphisms between plane groups, Bull. Amer. Math. Soc. 71 (1965), 629-630. MR 31 \#1374.

3. W. Magnus, Uber Automorphismen von Fundamentalgrupper berandeter Flachen, Math. Ann. 109 (1934), 617-646.

4. W. Magnus, A. Karrass and D. Solitar, Combinatorial group theory: Presentations of groups in terms of generators and relations, Pure and Appl. Math., vol. 13, Interscience, New York, 1966. MR 34 \# 7617.

Department of Mathematics, Princeton University, Princeton, New Jersey 08540

Department of Mathematics, University of Hawail, Honolulu, Hawail 96822

Current address (Joan S. Birman): Department of Mathematics, Stevens Institute, Hoboken, New Jersey 07030

Current address (Hugh M. Holden): Department of Mathematics, University of Hawaii, Honolulu, Hawaii 96822 